\title{
Upper Endoscopic Findings in Chronic Kidney Disease Patients
}

\author{
El-zahraa K. Mouhamed (resident), Amal K. Ahmed (MD), Eman A. Sabet (MD), \\ Lotfy H. Abo Dahab (MD). \\ Internal Medicine Department, Faculty of Medicine, Sohag University.
}

\begin{abstract}
Background: Patients with End stage renal disease (ESRD) suffer from recurrent gastrointestinal bleeding episodes with superficial mucosal inflammatory lesions. Lesions are more frequent in those who were in advanced stage of CKD and those undergoing dialysis.

Aim of the work: To determine upper endoscopic findings in CKD patients attending the Internal Medicine Department at Sohag University Hospital.

Patients and methods: The study included 70 patients (48 male ,22 females) with CKD who had upper GIT symptoms who were attending Internal Medicine Department or Dialysis Unit.

Results: The commonest upper endoscopic finding in CKD patients in our study is gastric erosion followed by atrophic pangastritis then peptic ulcer.

Conclusion: Gastrointestinal diseases are very common in patients of CKD, and it is easily documented with endoscopy. Some findings are more common than other, such as erosion, atrophic pan gastritis ,ulcer and reflux oesophagitis.
\end{abstract}

\section{Introduction}

Patients with End stage renal disease (ESRD) suffer from recurrent gastrointestinal bleeding episodes with superficial mucosal inflammatory lesions( Goyal M,et al 2014). Lesions are more frequent in those who were in advanced stage of CKD and those undergoing dialysis, reflecting a positive correlation of upper gastrointestinal lesions with the severity of CKD (Ahmed W, et al 2013). Gastrointestinal bleeding occurs with greater frequency and higher mortality in uremic patients than in the general population(Eiser AR ,2008).

It was reported that many abnormalities could be seen in upper endoscopy e.g erosions, ulcers, atrophic gastroduodenal folds, pale mucosa and moniliasis (Ahmed et al, 2003).

Aim of the study
To determine upper endoscopic findings in CKD patients attending the Internal Medicine Department at Sohag University Hospital.

To compare GIT symptoms and upper endoscopic findings in CKD patients on conservative treatment and CKD patients on dialysis.

\section{patients and methods}

The study included 70 patients (48 male ,22 females) with CKD who had upper GIT symptoms who were attending Internal Medicine Department or Dialysis Unit at Sohag University Hospital and patients were grouped into two main groups: -CKD on dialysis. CKD on conservative treatment The study was performed in two parts:

1. Retrospective part: revision of clinical, laboratory and endoscopic findings of patients with CKD who underwent upper endoscopic examination in the 
endoscopy unit of the department of Internal Medicine during the period from March 2015 to May 2016 (18 patients).

2. Prospective part: patients with CKD who were complaining from upper GIT symptoms and referred for upper endoscopic examination during the period from June 1016 to March 2017(52 patients).

For All patients, the following data were obtained:
1. $\mathrm{CKD}$ stage according to $\mathrm{K} / \mathrm{DIGO}$ criteria

2. GIT symptoms e.g anorexia, nausea, hiccough, bleeding and dyspepsia.

3. Drug intake e.g NSAIDs and steroids.

4. Co-morbidities e.g. Diabetes Mellitus, Liver disease,..

5. Routine investigations including $\mathrm{CBC}$, urine analysis and tests for liver functions

6. Upper endoscopy

7. Abdominal ultrasonography

\section{Results}

-The study was performed in Internal Medicine Department at Sohag University Hospital in the period from March 2015 to March 2017and included 70 patient (22 female \&48 male) who were complaining from upper gastrointestinal symptoms(GIT) necessating upper endoscopy .

-According to creatnine clearance study population were divided into two groups shown in

\begin{tabular}{|l||l|}
\hline CKD & Number (\%) \\
\hline \hline Conservative & $26(37.14 \%)$ \\
On dialysis & $44(62.86 \%)$ \\
\hline
\end{tabular}

Symptomatology :--

Patients were presented with different symptoms either separately or in combination frequency of upper GIT symptoms in study population

\begin{tabular}{|l|l|}
\hline Upper GIT symptoms & Number $(\%)$ \\
\hline Anorexia & $45(64.29 \%)$ \\
\hline Nausea & $41(58.57 \%)$ \\
\hline Vomiting & $29(41.43 \%)$ \\
\hline Dyspepsia & $25(35.71 \%)$ \\
\hline Hematemesis & $\mathbf{2 4}(34.29 \%)$ \\
\hline Hiccough & $\mathbf{2 1 ( 3 0 . 0 0 \% )}$ \\
\hline Melena & $16(22.86 \%)$ \\
\hline
\end{tabular}




\section{frequencey of upper GIT symptoms}

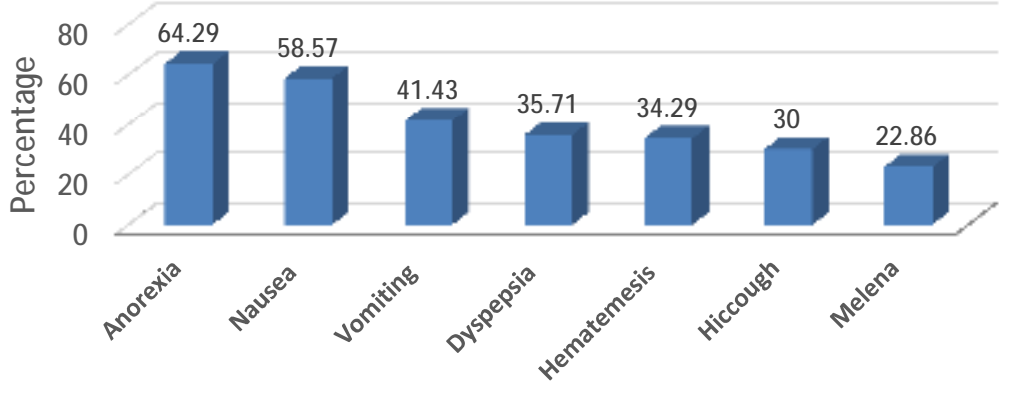

Upper endoscopic findings in patients with CDK on conservative treatment and those on dialysis

\begin{tabular}{|c|c|c|c|}
\hline & $\begin{array}{l}\text { Conservative } \\
\mathrm{N}=26\end{array}$ & $\begin{array}{l}\text { Dialysis } \\
N=44\end{array}$ & $P$ value \\
\hline Erosion & $12(46.15 \%)$ & $15(34.09 \%)$ & 0.32 \\
\hline Atrophicpan gastritis & $15(57.69 \%)$ & $11(25.00 \%)$ & 0.006 \\
\hline Ulcer & $5(19.23 \%)$ & $17(38.64 \%)$ & 0.09 \\
\hline Reflux oesophagitis & $5(19.23 \%)$ & $9(20.45 \%)$ & 0.90 \\
\hline PHG & $4(15.38 \%)$ & $6(13.64 \%)$ & 0.84 \\
\hline Varices & $3(11.54 \%)$ & $5(11.36 \%)$ & 1.00 \\
\hline Angiosysplesia & $1(3.85 \%)$ & 0 & 0.37 \\
\hline Bulbar doudenitis & 0 & $1(2.27 \%)$ & 0.44 \\
\hline
\end{tabular}

Upper endoscopic findings in patients with CDK on conservative treatment and those on dialysis

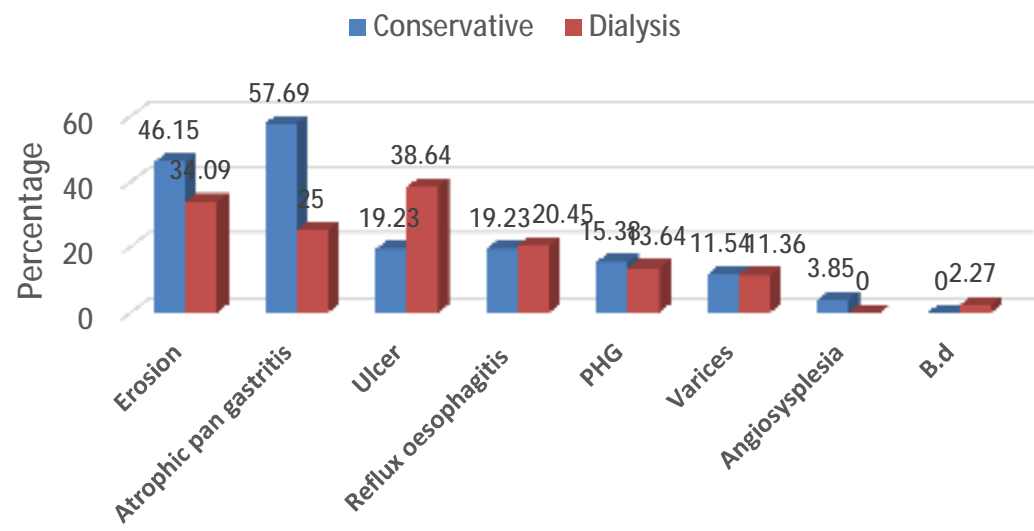




\section{Discussion}

In our study the The frequency of different GIT symptoms either separately or in combination was as the following :-the most frequent symptom was anorexia 45(64.29\%) followed by nausea $\quad 41 \quad(58.57 \%)$, vomiting 29(41.43\%),dyspepsia $25(35.71 \%)$,heamatemesis

$24(34.29 \%)$,hiccough 21(30\%) and melena $16(22.86 \%)$.

In our study ,although the frequency of symptoms was higher in patients on dialysis in comparison to those on conservative treatment the diffeerance was in significant except in heamatemesis ( $\mathrm{P}$ value $=0.002$ ) .

Similarly a study was performed in Kasr EL-Aini Hospital by Adel El-Sway, et al (2001) had found no significant difference in GIT symptoms between patients on conservative treatment and those on dialysis .

Endoscopic abnormalities were observed in all our patients. the most frequent abnormality was erosion(38.57\%) followed by atrophic pan gastritis (37.14\%) followed by ulcer(31.43\%) then reflux oesophagitis (20\%) followed by PHG (14.29\%) then by oesophageal varices $(11.43 \%)$ then by angiodysplesia $(1.43 \%)$ and bulbar doudenitis (1.43\%).Each endoscopic finding was found either separately or in combination.
In our study, we did not found significant difference in endoscopic finding between patients on dialysis and on conservative therapy .

On the other hand Adel El-Sway observed significant variation between CKD patients on dialysis and patients on conservative treatment as regard endoscopic abnormalities .

\section{Conclusion}

Gastrointestinal diseases are very common in patients of CKD, and it is easily documented with endoscopy. Some findings are more common than other, such as erosion, atrophic pan gastritis ,ulcer and reflux oesophagitis.

\section{References}

1. Goyal M, Charan S, Singh S, et al (2014). Study Of Upper Gastrointestinal Changes In Chronic Kidney Disease. International Journal of Bioassays. 30;3(11):3526-31.

2. Ahmed W, Qureshi H, Zuberi SJ, et al., (2013). Endoscopic lesions in chronic renal failure. JPMA J Pak Med Assoc; 43 (5): 95-6.

3. Eiser AR (2008). Gastrointestinal bleeding in maintenance dialysis patients. Semin Dial; 1: 198-202

4. Adel El-Sawy, Salah Z, El-Sersawy ,et al (2001) Upper gastrointestinal endoscopic findings in chronic renal failure hemodialysis versus conservative treatment 